\title{
LA INFLUENCIA DEL PAPADO DE AVIÑÓN SOBRE LA MÚSICA MEDIEVAL ESPAÑOLA: LA APARICIÓN DEL ESTILO ARS SUBTILIOR
}

\author{
ENIKŐ PAJOR
}

Universidad de Szeged

\begin{abstract}
I am not a musicologist but for more than twenty years I bave been regularly cultivating Gregorian chant. In the same way, personally I feel very close to medieval music. Preparing a training course for librarians specialized in music, I found a style called "Ars Subtilior" which is generally little known even among musicians, as it only lasted a few decades in the Middle Ages in France, Spain and Italy. I consider it important to provide some bistorical and theoretical data needed to understand the importance of this revolutionary new style in its historical context.
\end{abstract}

\section{Introducción}

"La muisica es algo más que un fenómeno estético: es una gnosis, una auténtica via de conocimiento..."

(Eugenio Trias)

El período del que voy a hablar transcurre durante los siglos XIII-XIV y dura aproximadamente sesenta años, sólo el tiempo de una generación, pero suficiente para que unos artistas, teóricos y músicos eminentes revolucionaran la notación, la estructuración y la representación de la música. Su esfuerzo intelectual y artístico perdura hasta hoy y se refleja en la música de los siglos XX-XXI.

La siguiente cita, relacionada con esta época, me ha dado un punto de vista diferente en la percepción de la misma, ayudándome a sentar las bases de este estudio. Una de las revistas musicológicas húngaras, La Música publicó una entrevista con el compositor György Ligeti (1923-2006), quien, hablando de su obra titulada Estudios para piano, afirmó: "... el estilo de Palestrina estaba dado [...], pero conocia muy poco de la misica anterior, y cuando interpretaba los temas de Okbegem sólo para mi, todo un mundo se abria ante mis ojos: me di cuenta de que babia un tipo diferente de polifonia a la de Palestrina. Por eso, en mis Estudios para piano en vez de Okbegem se observa la influencia de una época anterior, la del Ars Subtilior [...]." 1

\footnotetext{
${ }^{1}$ András VARGA BÁLINT, Mária FEUER, “A komponálás nem akarat kérdése. Születésnapi beszélgetés Ligeti Györggyel" [La composición no es una cuestión de valentia. Una entrevista de
} 


\section{Antecedentes históricos}

"Las composiciones del Ars Nova siguen pareciendo sorprendentes hoy en dia por su modernidad y audacias ritmicas." (Sydney d'Agvilo)

La música del Ars Nova nace en siglo XIV. Comienza con las primeras décadas del siglo e incluye las siguientes (1320-1380). Durante este tiempo en Francia trabajaban generaciones sucesivas de dos compositores, mientras que en Italia el compositor Francesco Landini (c. 1325 - c. 1397) ya trabajaba como la tercera generación de compositores de este estilo, conocidos hoy en día como "generación de la música del Trecento".

El papado de Aviñón (1309-1377) fue el centro de este movimiento y tuvo un papel importante sobre las artes en el sur de Francia, norte de la Península Ibérica y de Italia y en todas las cortes aristocráticas donde la cultura había sobrevivido al influjo intelectual de Aviñón. Desde el punto de vista del arte musical, el reino de Aragón fue uno de los principales beneficiarios de que la residencia papal se estableciera en Aviñon. Tanto el Papa como sus cardenales contaban con cantores a sus servicios y atraían a los compositores más famosos de la época. "En Aviñón trabajaron algunos de los compositores más afamados de su tiempo, la mayoria chantres que cruzaron en múltiples ocasiones las fronteras del reino aragonés con el propósito de ingresar en alguna de las capillas que mantenía la Casa Real. [...] Al principio, y salvo excepción, los juglares eran los españoles y los ministriles de los extranjeros, en su mayoria franceses y flamencos que acudian de visita a las casas de la nobleza local." "El reino de Aragón hizo grandes esfuerzos para llegar a ser uno de los promotores de la música europea. Gracias a su proximidad con Francia y a las bodas de algunos de sus reyes con damas francesas -como hizo Juan I-, recibió una gran influencia de las costumbres culturales francesas. ${ }^{3}$ Sobre todo la música, cuyas nuevas corrientes, como el Ars Nova, penetraton en la península de la mano de los músicos ambulantes que desde otros países llegaban buscando fortuna en las cortes y los señoríos. La música hispánica de la corte comenzó a tener éxito a partir del último periodo del siglo XIV.

cumpleaños con el compositor György Liget], in: Muzsika, 41, 1998., núm. 5, 3. Accesible en http://epa.oszk.hu/00800/00835/00005/1613.html, fecha de consulta: el 22 de junio de 2012.

2 María del Carmen GÓMEZ MUNTANÉ, La música medieval en España. Madrid, Kassel Reichenberger, 2001, XVI, 219-220, 239, 274.

3 Samuel RUBIO, Historia de la muisica española 2. Desde el "ars nova" basta 1600, Madrid, Alianza Editorial, 2006, 106-109. María del Carmen GÓMEZ MUNTANÉ, La muisica en la casa real cataloaragonesa, 1336-1432. Vol. I, Historia y documentos, Barcelona, 1979. 


\section{Percepciones de la música}

'En la música, como en todo arte, bay también algo de ciencia; y asi como el pintor, pongamos por caso, necesita de ciencias auxiliares como la geometría, la matemática...."

(Palabras de Sagastume Berra de los fundamentos matemáticos de la música)

Desde la Antigüedad los filósofos se han hecho estas preguntas: ¿la música es un arte o una ciencia? ¿La ciencia es un arte también? He aquí una respuesta: "Cuando la práctica de las artes y de las ciencias alcanza su nivel más alto o major esplendor, es aspiración común de ambas el querer convertirse la una en la otra." "La mayoría de los filósofos coinciden en que la práctica de la música a un alto nivel la convierte en ciencia. No se trata solamente de una práctica superior, virtuosa; en la creación, en la composición musical, en su estructura, podemos descubrir elementos científicos. El fenómeno más prominente es la relación entre la música y las matemáticas. Cuando se estableció la doctrina de las artes liberales, entre las que se encontraba la música, Boetio, en su obra titulada De instituciones de música, trató de una versión libre de las obras correspondientes de los pitagóricos Nicómaco de Garesa y de Ptolemao, de forma que el pensamiento musical griego -en su interpretación más matemática- llegaria a occidente a través de este tratado 'La vinculación de Música y Matemáticas en el curriculum dio a la teoria musical una dimensión cientifica." 5

\subsection{Formas inmensurables y mensurables}

'Ningún arte se constituye sin proporción; y la proporción reside en el número. Asi pues, todo arte se constituye por medio del número...

$Y$ es razonable decir que «todo se parece al numero», es decir, a la razón capaz de juggary afin a los numeros que componen todas las cosas. Eso dicen los Pitagóricos." (Tatarkienric:: Textos pitagóricos, Sexto Empirico, Adv. Mathem. VII, 106.)

En general, todo arte es un sistema de percepciones. Ya en la Antigüedad los filósofos y los estetas examinaron las obras artísticas musicales desde un punto de vista especial: en función de si podían ser representadas y explicadas matemáticamente o no. Esta es la ciencia de la estética del número. Se distinguía entre aquellas formas susceptibles de ser representadas matemáticamente, las

\footnotetext{
${ }^{4}$ Sydney d'A GVILO, Epistemologia de la misica, Madrid, Intervalic University, 2001, 189-190.

${ }^{5}$ Nadine HENRARD-Paula MORENO-Martine THIRY-STASSIN (eds.), Convergences médiérales: épopée, lyrique, roman, Bruxelles, De Boeck Université, 2001, 29. Véase también Carlos ALVAR, Traducciones $y$ traductores: materiales para una bistoria de la traducción en Castilla durante la Edad Media, Madrid, Centro Estudios Cervantinos, 2010, 131.
} 
"formas mensurables", de aquellas otras que no eran accesibles a una organización y representación matemática, las formas inmensurables. Queda patente que la tradición vincula el significado de los números con el efecto harmónico, perceptible principalmente en la música.

En suelo ibérico un representante de esta corriente de pensamiento fue el francés Johannes de Muris (1290-1351), eclesiástico filósofo, matemático y astrónomo, quien el 14 de mayo de 1333, en presencia de la reina Juana II de Navarra (1312-1349), observó un eclipse sobre el cual había constatado unos elementos desconocidos, nuevos. A partir de entonces, De Muris, por el que también se había interesado el papa Clemente VI (1291-1352), empezó a trabajar regularmente en la corte de ambos mandatarios, aficionados, por otro lado, a la música. La Casa de Navarra no tardó en seguir el ejemplo de la aragonesa para invitar a músicos famosos. ${ }^{6}$ De Muris, como músico, cultivó la música especulativa, sobre la que publicó cuatro libros. ${ }^{7}$ Entre ellos, el más conocido es su Libellus cantus mensurabilis, en el que aplicó las proporciones numéricas a la música polifónica. Su teoría se apoya en la idea de que existen relaciones entre las notas, las proporciones numéricas de la música y las del universo. ${ }^{8}$

En esta época, como ya se ha dicho, los franceses Philippe de Vitry (12911361) y Johannes de Muris (c.1290-c.1350) fueron los más importantes teóricos de la música mensurable.
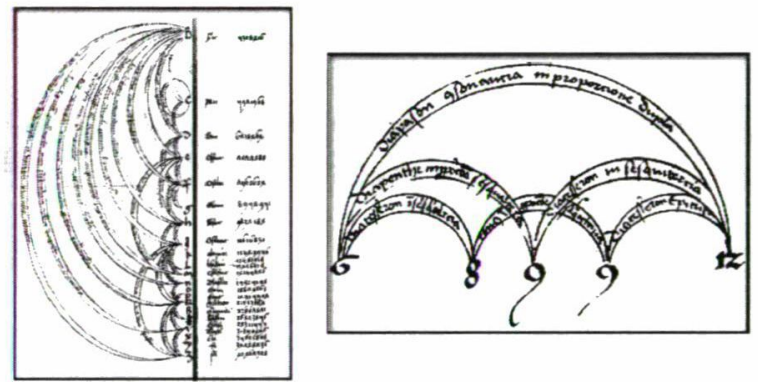

Figuras 1-2. Johannes de MURIS: Libellus Cantus Mensurabilis ${ }^{9}$

${ }^{6}$ GÓMEZ MUNTANÉ, op. cit., 219.

${ }^{7}$ Estos son: Ars Novae musicae (1319), Musica speculativa secundum Boetbium (1323), Beellus cantus mensurabilis (1340-1350, reprise de Philippe de Vitry), "De sonis musicis" in: Quadripartitum opus numerorum (1343).

${ }^{8}$ Enrico FABINI, La estética musical desde la Antiguiedad basta el siglo XX, Madrid, Alianza, 1992. El trabajo de Muris para la unificación del quadrivium constituyó una parte importante del plan de estudios universitarios de la Sorbona -donde había estudiado y más tarde fue profesor-, durante los siglos XIV-XV.

9 Ms. H 165. inf., Biblioteca Ambrosiana, 1499, red. por Gafurio, f. 1r-15v, Ars novae; f. 16v$18 \mathrm{v}$, Glossemata super [...] (attrib.); f. 18v-22v. 


\subsection{El sistema rítmico-modus, métrica grecolatina, notación mensural}

\section{"... lo cierto es que la investigación bistórica de la notación mensural.}

se ba ocupado principalmente del estudio de los elementos objetivos cuantificables o racionales: métrica, cantidad, aritmética. (Anuario Musical, 2007.)

Aunque el papel de la música era secundario en comparación a otras artes, a principios del siglo XIII se convirtió en objeto de contemplación de eruditos y teóricos, gozando de la misma importancia que en la Antigüedad. Lo más importante era la problemática de la notación musical, particularmente "la notación mensural", que usaba formas de notación individuales para denotar las duraciones temporales. El desarrollo más profundo de "la notación mensural" reside en la capacidad de este sistema para indicar ritmos complejos con gran exactitud y flexibilidad. ¿Por qué tenía tanta importancia este fenómeno? Porque hasta 1280 la representación o notación musical utilizó el sistema "ritmomodus", basado en los pies métricos de la poesía clásica. La métrica de los versos en la Antigüedad utilizaba la oposición de duración de silabas largas y breves, que se combinaban dando lugar a distintos patrones o pies. Fue la famosa Escuela de Nôtre-Dame donde comenzaron a aplicarse a la música estos esquemas rítmicos de la métrica clásica griega, de forma que se diferenciaban dos valores básicos: largo y breve, en el que el largo era el doble que el breve, de forma que la suma de ambos era tres. ${ }^{10}$ En la Edad Media, los cantantes interpretaban fundamentalmente obras de canto gregoriano, cuyo ritmo seguía el propio de la letra; de ahí que para ellos la utilización del "sistema ritmomodus" era algo natural. Como sabemos por la descripción de Rescigno R. Garagavlia, para un cantante era suficiente ver las palabras "Verbum Patris humanator", por ejemplo, para saber cantarlas como un verso trocaico. Pero esta forma de notación cambió completamente a raíz de que el teórico musical Franco de Colonia (Franco Teutonius) (c. 1215?-1270?), introdujera en su obra Ars cantus mensurabilis (1260) su sistema de "notación mensural", hoy en día también conocido como sistema de "notación franconiana", gracias al cual ya no era necesario conocer el "modus" antiguo. En este sistema, la duración de un sonido es determinable por la grafia utilizada para anotarlo, independientemente del contexto musical en que se encuentre: músicos y matemáticos habían inventado una forma de puntuación cuya lectura no necesitaba del conocimiento

${ }^{10}$ Los modos rítmicos eran: Troqueo: largo-corto; Yambo: corto-largo; Dáctilo: largo-cortocorto; Anapesto: corto-corto-largo; Espondeo: largo-largo; Tribraco: corto-corto-corto. 
del "modus" del ritmo clásico. A partir de este momento el tempo en la música no estaba determinado por la poesía antigua, sino por esta métrica. A pesar de todo el desarrollo y perfeccionamiento de la ley del "tempo simbólico" supuso toda una revolución en la vida intelectual, musical y artística en general de la Edad Media." Poco a poco, este peculiar método de composición musical se puso de moda, permitiendo crear una música más sensible y sofisticada que las anteriores. Los compositores que lo adoptaron llamaban a los que seguían la tradición "oscurantistas" y, oponiéndose a ellos, crearon nuevos géneros y procedimientos que dieron lugar al Ars Nova. Este estilo derivó en una forma más manierista, conocida como Ars Subtilior, la cual apenas se cultivó durante unas décadas, pero que sin embargo revolucionó completamente la notación y la composición musicales, y en consecuencia, la interpretación de la música.

\subsection{Géneros, formas, características compositivas}

"No se puede realmente llegar a comprender los géneros musicales de la Edad Media sin tener en cuenta dos aspectos esenciales: por una parte, el peso que tenia

La Iglesia en la construcción de la realidad de aquel tiempo; por otra parte, la necesidad que tenia esa Iglesia de propagar el mensaje evangélico por todos los canales a su alcance..." (Gérard Denizeau)

Gracias a la carismática personalidad de los compositores del Ars Nova / Ars Subtilior, la profesión ganó prestigio y se puedo independizarse de los talleres eclesiásticos, sobre todo en Francia. La música comenzó a separarse de la música eclesiástica y de la liturgia, aunque los géneros musicales seguían siendo los mismos (motetes, ordinarios, baladas, rondós, virelais). Lo que era nuevo es la manera de abordar su representación, la utilización compleja e insólita de varios elementos musicales como por ejemplo los elementos siguientes:

- Ritmos complejos -en el solo cantus interpretado por el solista y en el resto-. Son frecuentes las series de sincopa, las pausas intermitentes en las frases musicales, los diversos cambios de las divisiones o intervalos perfectos $\mathrm{e}$ imperfectos. Da la sensación de que los compositores quisietan reflejar la interpretación libre (en rubato) del cantante.

- Las composiciones monódicas cambiaron y la polifonía apareció con una melodía previa que servía de base, llamada cantus firmus, donde los solos de contratenor y tenor a menudo se cruzaban, pero el equilibrio armónico de base no

11 Géza SZAMOSI, “A polifón zene és a klasszikus fizika. A newtoni időfogalom eredete." [La música polifónica y la fisica clásica: los origenes del concepto de tiempo de Newton], Fizikai Szemle, 1991, núm. 8. Accesible en: http://wrww.kfhi.hu/fszemle/archivum/fsz9108/szg9108.html, fecha de consulta: el 30 de julio de 2012. 
desapareció. $^{12}$ Podemos considerar que, en origen, la polifonia era un tropo musical que en vez de añadirse de forma sucesiva a una obra gregoriana, se añadía de forma simultánea. De esta manera comenzó a desarrollarse un interés por la relación vertical (armónica) de las voces. La aparición de la polifonía es uno de los factores que propició la aparición de la notación mensural, permitiendo diferenciar tanto la altura de los sonidos, como la duración de las notas. ${ }^{13}$

\subsubsection{Macroformas, microformas, formas especiales con notas coloreadas}

'Los compositores inventaron un modo de representación de la música absolutamente original porque no eligieron un sistema: bicieron converger todos los que babia."

La representación y descripción de la música estaba subordinada a la Iglesia. En la estructura, en el texto, en la notación reconocible los elementos de las obras filosóficas, epistemológicas se escriben como macro- y microformas. El ritmo ternario se representaba con un circulo, símbolo de la perfección. Era conocido como ritmo perfecto en homenaje a la Santísima Trinidad. Más tarde este sistema se complicó aún más, hasta dar origen a nuestra notación actual. Cuando nos refiramos a la forma global de la obra musical, o a una obra del arte, la denominaremos macroforma. Por el contrario, cuando la estudiemos en detalle, o de "la manera en que las fibras de tejido se entrelazan para formar un tipo de disposición artística", nos estaremos refiriendo a la microforma. La distinción entre la macroforma y la microforma deriva de la antiquísima separación entre las escalas perceptuales del macrocosmos -el Universo-, y del microcosmos -el Hombre-, que aparece ya en los tratados de filosofía de la Antigüedad. ${ }^{14}$ En la notación mensural de las obras, la complejidad del ritmo, de las alteraciones y cambios de representación requieren el uso frecuente de notas de color rojo. Las transcripciones y notaciones a veces también expresaban con sus formas o disposiciones espaciales el contenido del texto. Entre ellos encontramos formas sorprendentes, inhabituales, es decir, por ejemplo, corazones y composiciones en forma de arpa. La forma circular es también utilizada frecuentemente para los cánones con valor de doble símbolo: el canon

${ }^{12} \mathrm{La}$ música polifónica "se seguía componiendo a dos o tres voces (cantus firmus $y$ duplum 0 también triplum), pero la intenvención de la cuarta era cada wez más frecuente. Esta voz era inferior al cantus firmus y poseia valores del mismo orden de duración respecto a la voz principal. En Francia se le denominó contre-teneur, quedando asi constituido el cuarteto vocal, considerada la formza polifónica ideal hasta principios del siglo XVII". Véase en http://es.wikipedia.org/wiki/Ars_nova, fecha de consulta: el 30 de julio de 2012.

${ }^{13}$ Largo, breve, semibreve etc. El semibreve será el valor de referencia equivalente a nuestra redonda actual.

14 Sydney d'AGVILO, Morfologia del arte. Epistemologia de la forma artistica, Madrid, Intervalic University, 2002, 89. 
puede repetirse hasta el infinito, y la forma circular se refiere la eternidad infinita de Dios. Así es como la música, el arte y la fe estaban vinculados. En todos estos modos de representación característicos de esta edad no es difícil descubrir la artificialidad del esfuerzo. Esto explica el significado del término Ars Subtilior, originalmente 'notación amanerada, estilo manierista'. ${ }^{15}$

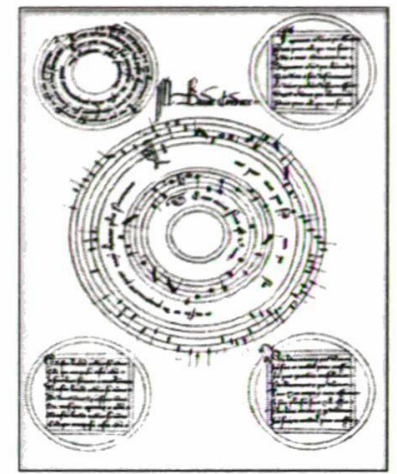

Figura 3. El canon circular de Baude Cordier (c.1380 - c.1440) $)^{16}$

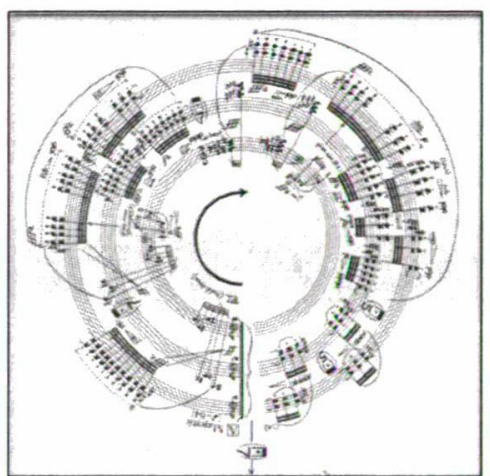

Figure 4. Una parte del canon círcular de George Crumb (1929-) ${ }^{17}$

${ }^{15}$ A partir de los años 1960 es nombrado Ars Subtilior por la musicóloga alemana Ursula Günther para evitar las connotaciones negativas de los términos anteriores. Véase Ursula GÜNTHER, "Das Ende der Ars Nova", Die Musifforschung, 16, 1963, 105-120 y también Willi APEL, The notation of polpphonic music, 900-1600, Cambridge, Mass, The Mediaeval academy of America, 1942.

${ }_{16}$ Fuente: http://images-mediawiki-sites.thefullwiki.org/07/3/5/3/28351073512308670.gif, fecha de consulta: el 14 de agosto de 2012.

${ }^{17}$ Fuente: http://www.panopticist.com/2006/02/downward_movement.php, fecha de consulta: el 14 de agosto de /2012. 


\subsection{Música colorata, música falsa, música ficta}

"Sine falsa música non possimus discansare" "Falsa musica est, quando de tono facimus semitonium et converso"

(Anónimos)

También en esta época nació una nueva práctica, en la que hay una gran diferencia entre la notación y la interpretación. Aquí no tenemos la posibilidad de explicar el sistema hexacordal con su escala diatónica, del cual trata el libro De speculatione musica de Walter Odington (1316), pero haremos un pequeño apunte. El autor sugiere a los músicos la ejecución de sonidos no escritos, es decir, de sonidos que no aparecen en la partitura, cuando encontraran un "signo colorato". Así apareció el fenómeno de la "música falsa", inusitada, colorata o conjunta. El término más utilizado en los siglos XIII-XIV fue el de música ficta. El término ficta quiere decir 'fingida' o 'imaginada', en el sentido de que hay que imaginar notas alternativas a las que están escritas. Según los músicos de la época, la música ficta era necesaria doblemente para "conocer la perfección de las consonancias imperfectas y de las disonancias y una resonancia más dulce que la armonia." 18

Este tipo de composición, con notas coloradas para indicar la alteración de los valores y formas inhabituales de representación en función del sentido del texto formas de corazón, varios círculos etc. -, llegó a tener mucho éxito y la Iglesia lo utilizó con tan excesiva permisividad, que en 1325 el Papa Juan XXII (1249$1334)^{19}$ emitió una bula que condenaba las prácticas de aquellas escuelas musicales donde los alumnos utilizaban con gran diligencia las nuevas formas de medida de tiempo. Aparte del Papa, los representantes del estilo anterior, es decir, del ars antiqua, habían protestado también: "[Los modernos] ... tienen poco respeto por los antiguos maestros, sus predecesores. Rechazan con bechos (pese a lo que puedan decir con sus palabras) la buena doctrina por ellos enseñada, cambiándola en algunas partes, corrompiéndola, reprobándola, anulándola [...] usan un nuevo modo de cantar y abandonan el antiguo, bacen un uso excesivo de las medidas imperfectas, tienen afición por las notas semibreves, que llaman minimas, y rechazan las composiciones antiguas: organa, conductus, motetes, boguetus a dos y a tres voces; [...] componen discantus sutiles $y$ difíiciles de cantar $y$ 'de medir". ${ }^{20}$ Pero la citada bula no disuadió a nadie, y mucho menos al filósofo, matemático, compositor y

\footnotetext{
18 Paloma OTAOLA GONZÁLEZ, Tradición y modernidad en los escritos musicales de Juan Bermudo. Del Libro primem (1549) a la Declaración de instrumentos musicales (1555). Madrid, Kassel, Reichenberger, 2000. 126-131.

${ }^{19}$ Papa núm. 196 de la Iglesia católica, de 1316 a 1334.

20 Accesible en: http://usuarios.multimania.es/grupocantofigurado/estudios.html, fecha de consulta: el 30 de julio de 2012.
} 
teórico de la música Philip de Vitry (1291-1361), que en 1322 escribió una obra titulada Ars Nova (1322), la cual sienta las bases teóricas de este arte. ${ }^{21}$

Entre las personalidades más destacadas del Ars Subtilior que trabajaron al servicio de Leonor de Aragón (1358-1382), reina consorte de Castilla ${ }^{22}$ por su matrimonio con Juan I de Castilla (1358-1390), encontramos al famoso Jacob Senleches (c.1378-1395), un compositor francés que trabajó después como arpista para el cardenal Pedro de Luna (1328-1423), más tarde elegido papa de Aviñón con el nombre de Benedicto XIII o el "Papa Luna" (1394). ${ }^{23}$ Senleches había desarrollado muchas innovaciones rítmicas y notacionales. Su obra más conocida es La barpe de mélodie, "que tiene dos voces superiores en canon, con figuraciones irregulares, y un tenor supuestamente instrumental. La pieza se escribió en forma de arpa, utilizando las cuerdas de ésta como lineas para escribir las notas, complementando asi la complejidad de la composición con la de la notación." ${ }^{24}$

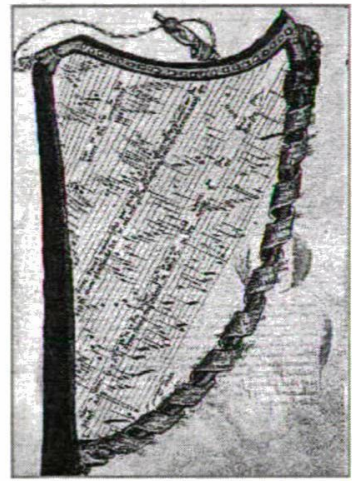

Figura 5. La obra más conocida de Jacob de Senleches: La harpe de mélodie, dibujada en el estilo Ars Subtilior ${ }^{25}$

\footnotetext{
${ }^{21}$ Esta obra presenta la práctica totalidad de las innovaciones teóricas. Principalmente el método mensural y ritmo y el desarrollo del concepto de isoritmia.

${ }^{22}$ A la muerte de la reina, el 15 de septiembre de 1382, Senleches compuso la balada titulada Fuions de ci (Huyamos de aquî) y pasó después al servicio del cardenal Pedro de Luna. Véase María Carmen GÓMEZ, "Musique dans les Chapelles de La Maison Royale d'Aragon (13361413)", in: Música Disciplina, vol. 38, 1984, 72.

23 Ursula GÜNTHER, "Zur Biographie Einiger Komponisten der Ars Subtilior", Archiv für Musilewissenschaft, vol. 21, 1964, 197. Véase también Jason STOESSEL, The Captive Scribe: The context and culture of scribal and notational process in the music of the ars subtilior. Manuscrito, tesis doctoral. Armidale, Australia, University of New England, 2002. Accesible en: http://www.diamm.ac.uk/redist/pdf /Stoessel_v1.pdf, fecha de consulta: el 30 de septiembre de 2012.

${ }^{24}$ Accesible en: http://es.wikipedia.org/wiki/Jacob_Senleches, fecha de consulta: el 30 de julio de 2012.

${ }^{25}$ Fuente accesible en formato MP3: http://www.youtube.com/watch?v=9RBtL_-VpWs, fecha de consulta: el 30 de septiembre de 2012.
} 


\section{Códices y manuscritos franceses e hispánicos que guardan elementos del Ats Nova y del Ars Subtilior}

Como afirma Samuel Rubio, hasta ahora no se conocen documentación histórica ni obras musicales que nos den testimonio de la existencia de centros importantes castellanos en los cuales se cultivara la música del Ars Nova. ${ }^{26} \mathrm{La}$ mayoría de los manuscritos son franceses e italianos. M. C. Gómez Muntané, en su libro escrito sobre la música en la Casa Real catalano-aragonesa ${ }^{27}$ ha recogido y transcrito todos los manuscritos y fragmentos conservados en las bibliotecas catalanas.

\subsection{El Llibre vermell o Libro rojo}

De esta época encontramos un códice singular, el Llibre vermell o Libro rojo ${ }^{28}$, que se guarda en el monasterio de Montserrat, cerca de Barcelona. Este códice contiene piezas polifónicas a dos y tres voces con notas coloreadas también para a los devotos que venían al santuario de la Virgen en peregrinación. Lo interesante es que mientras en Francia en esta época ya existían obras polifónicas en lengua vulgar o cantos profanos, en la Península Ibérica únicamente encontramos este códice, que tiene una temática general religiosa cuyo marco no es obligatoriamente la Iglesia.



Figura 6. El verso del folio XXII del Llibre vermell 29

\footnotetext{
${ }^{26}$ Samuel RUBIO, op. cit., 107.

${ }^{27}$ María del Carmen GÓMEZ MUNTANÉ, La música en la Casa Real catalano-aragonesa durante los años 1336-1342, I. Michigan, Bosch, 1979 (digitalizado en 2007), 233.

${ }^{28}$ Se llama así por el color de su encuadernación.

29 Fuente: http://www.cervantesvirtual.com/obra-visor/llibre-vermell-de-montserrat-- $0 / \mathrm{html}$ /ff6fe3e2-82b1-11df-acc7-00 fecha de consulta: el 17 de agosto de 2012.
} 


\subsection{El Roman de Fauvel}

Entre los manuscritos del Ars Nova / Ars Subtilior, el más famoso es el $\mathrm{N}^{\circ} 146$ de la Biblioteca Nacional de París, Le Roman de Fauvel, una sátira alegórica de la Iglesia católica y del Estado de comienzos del siglo XIV, el cual usa la metáfora de un ambicioso burro que hace una peregrinación. En el camino se encuentra a varios personajes que representan la mentira, la corrupción, la hipocresía etc., es decir, todos los pecados del humanidad. En el fin del mundo Fauvel viaja al Macrocosmos esperando encontrarse con La Bondad. Pero alli la vida tampoco es mejor; sus habitantes son el Odio, la Pereza, la Gula, la Avaricia, la Mezquindad, el Orgullo etc. El nombre del asno, Fau-vel, significa 'mentira velada' y constituye un acróstico, ya que cada letra representa un pecado capital:

"Flaterie si s'en derive... (adulación)

Et puis en descent Avarice (avaricia)

Vileine (villanía, conciencia)

et Variété (veleidad, desvergüenza)

Et puis Envie (envidia)

et Lacheté..." (cobardía)



Figura 7. Motivos de las personajes del Roman de Fauvel 30

${ }^{30}$ Fuente: Libreto del CD del Roman de Fauvel por la Camerata Boston. Accesible en: http://bertdekkers.nl/klassiek/fauvel_list.html, fecha de consulta: el 30 de septiembre de 2012. 
La atención de los musicólogos está puesta en las 169 piezas musicales escritas en estilo Ars Aova / Ars Subtilior, interpoladas entre los 3.000 versos del texto. Aunque este no sea muy conocido, la música es interpretada y registrada regularmente en todo el mundo. Según los expertos, se trata de una importante antología de la música secular y religiosa realizada entre 1170 y $1316 .{ }^{31}$

\subsection{Codex de Chantilly (Chantilly, Museo Condé MS 564)}

En la ciudad francesa de Chantilly se halla guardado el manuscrito más famoso de la segunda mitad del siglo XIV, periodo en el que el estilo Ars Subtilior tuvo gran éxito tanto en Francia como en Italia y en la Península Ibérica. En el manuscrito se hallan 112 piezas polifónicas de diversos géneros de su tiempo. Entre ellas la más conocida es el rondó del compositor francés Baude Cordier (c. 1380-antes de 1440) Belle, bonne, sage, cuya notación representa alteraciones rítmicas con notas rojas y adopta la forma de un corazón para reforzar el sentido de su texto.
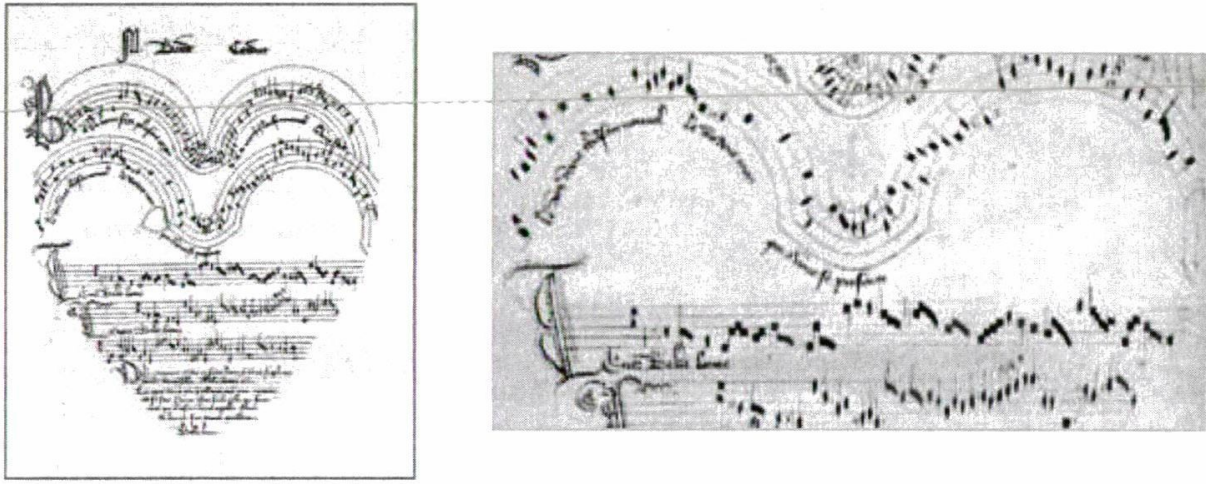

Figuras 8-9. Imagen completa y parcial del Rondó Belle, Bonne, Sage de Baude Cordier ${ }^{32}$

\footnotetext{
${ }^{31}$ Jesús J. LACASTA SERRANO, "Música y danza en las calles y plazuelas de la villa medieval", in: Jornadas de Canto Gregoriano, XIII. Música en la bispania romana, visigoda y medieval. Más allá del atrio de la iglesia y de la cerca del monasterio, Zaragoza, Institución Fernando el Católico (C.S.I.C.) / Excma. Diputación de Zaragoza, 2010, 69-109. Accesible en: http://ifc.dpz.es/recursos/publicaciones/30 /34/04lacasta.pdf, fecha de consulta: el 30 de julio de 2012; Texto entero del Roman de Faunel accesible en: http://www.archive.org/stream/leromandefauvel100gervuoft/, fecha de consulta: el 30 de septiembre de 2012. Bibliografia y discografia muy compleja, accesible en: http://www.arlima.net /eh/fauvel.html, fecha de consulta: el 30 de septiembre de 2012.

${ }^{32}$ Fuentes: http://www.paperblog.fr/1395728/baude-cordier-belle-bonne-sage-un-rondeau-au-xvesiecle-ii/, fecha de consulta: el 30 de septiembre de 2012, http://eeleach.wordpress.com/2010/05/ fecha de consulta: el 30 de septiembre de 2012.
} 


\section{Conclusión}

Como profesora-recuperadora de información de textos antiguos me interesa cada tema como objeto posible para una nueva búsqueda. Los temas raros, menos conocidos son especialmente adecuados para aplicar los conocimientos históricos y epistemológicos en la práctica. Por consiguiente, el gran resultado de este trabajo fue que mis estudiantes, futuros bibliotecarios musicales y al mismo tiempo músicos, no solamente estudiaran el uso de fuentes como la Red profunda/invisible o la Red semántica, sino que entendiesen que todavía hay formas y estilos musicales que pueden sobrevivir al paso de los siglos y volver a la actualidad. Un ejemplo de ello es la música de Paul Hindemith, de György Ligeti, y del pianista y creador de la nueva teoría musical "sistema interválico", el español Sydney d'Agvilo. 